\title{
Síntese in situ pelo método hidrotérmico de heteroestruturas de titanato-ferritas e sua atividade fotocatalítica
}

\section{(In situ hydrothermal synthesis of titanate-ferrite heterostructures and their photocatalytic activity)}

\author{
B.F.Troque ${ }^{1}$,S.A. Eliziário ${ }^{1}$, M.J. Godinho ${ }^{1,2}$, R. H. G.A. Kiminami ${ }^{1 *}$ \\ ${ }^{1}$ Universidade Federal de São Carlos, Departamento de Engenharia de Materiais, 13565-905, São Carlos, SP, Brasil \\ ${ }^{2}$ Universidade Federal de Goiás, Instituto de Física e Química, RC, Goiânia, GO, Brasil
}

\begin{abstract}
Resumo
A aplicação de fotocatalisadores na forma de heteroestruturas tem se destacado através da propriedade de degradação de diversos compostos orgânicos no tratamento de efluentes. Neste contexto, o objetivo deste trabalho foi sintetizar compostos heteroestruturados à base de titanato-ferritas pelo método hidrotérmico in situ para aplicação em processo foto-Fenton. Os materiais sintetizados in situ foram caracterizados por difratometria de raios X, espectroscopia de infravermelho, espectroscopia de reflectância difusa, área específica superficial por BET e microscopia eletrônica de transmissão. A síntese in situ de $\mathrm{SrTiO}_{3}-\mathrm{CoFe}_{2} \mathrm{O}_{4}$ e $\mathrm{SrTiO}_{3}-\mathrm{NiFe}_{2} \mathrm{O}_{4}$ foi desenvolvida de forma inédita neste trabalho, resultando com sucesso em sistemas heteroestruturados sem presença de fases secundárias, distribuição altamente homogênea das fases constituintes com tamanho médio de partículas nanométrico e garantia de reprodutibilidade do método. A atividade fotocatalítica dos compostos sintetizados foi investigada por meio da degradação do corante Rodamina B, utilizando luz visível, onde o sistema $\mathrm{SrTiO}_{3}-\mathrm{CoFe}_{2} \mathrm{O}_{4}$ apresentou a melhor atividade fotocatalítica degradando $82 \%$ do corante.
\end{abstract}

Palavras-chave: síntese, método hidrotermal, heteroestruturas, titanato de estrôncio, ferritas, fotocatálise.

\begin{abstract}
Heterostructured photocatalysts stand out for their ability to degrade various organic compounds in the treatment of wastewater. In this context, the purpose of this work was to produce titanate-ferrite heterostructured composites by in situ hydrothermal synthesis for application in the photo-Fenton process. The synthesized materials were characterized by X-ray diffraction, infrared spectroscopy, diffuse reflectance spectroscopy, specific surface area (by BET) and transmission electron microscopy. The in situ synthesis of $\mathrm{SrTiO}_{3}-\mathrm{CoFe}_{2} \mathrm{O}_{4}$ and $\mathrm{SrTiO}_{3}-\mathrm{NiFe}_{2} \mathrm{O}_{4}$ was developed here in a novel way, successfully resulting in heterostructured systems with no secondary phases and a highly homogeneous distribution of the constituent phases with average nanometric size particles. Moreover, this new method was found to be highly reproducible. The photocatalytic activity of the synthesized composites was analyzed based on the degradation of Rhodamine $\mathrm{B}$ dye using visible light. The best photocatalytic activity was exhibited by the $\mathrm{SrTiO}_{3}-\mathrm{CoFe}_{2} \mathrm{O}_{4}$ system, which degraded $82 \%$ of the dye.
\end{abstract}

Keywords: hydrothermal method, heterostructures, strontium titanate, ferrites, photocatalysis.

\section{INTRODUÇÃO}

Os processos de degradação para eliminação de poluentes orgânicos presentes na água, como corantes, herbicidas, pesticidas, resíduos de antibióticos, hormônios, dentre outros, representa hoje um grande desafio, já que o tratamento convencional, na maioria das vezes, não remove de forma eficiente esses compostos. A degradação de compostos orgânicos, como no caso de corantes,

*ruth@ufscar.br é de fundamental importância para não contaminar o ambiente aquático, já que, uma pequena quantidade de corante em água (10-20 mg/L) é altamente visível e afeta a transparência da água levando a um desequilíbrio do meio [1]. Uma das formas de tratamento desses compostos orgânicos é através de processos oxidativos avançados (POA), onde a fotocatálise heterogênea é uma das tecnologias mais promissoras para remediação ambiental e tratamento de efluentes em geral. Essa técnica consiste na excitação eletrônica de um semicondutor, denominado de fotocatalisador, de preferência pela luz 
solar [2]. A mineralização dos compostos orgânicos mediante fotocatálise por ação de um semicondutor iniciase quando o mesmo é fotoativado, resultando na geração do par elétron/buraco. A geração deste par é mais interessante quando proporcionada por radiação eletromagnética com comprimentos de onda no UV-vis, a qual leva à diminuição dos custos do processo. Uma alternativa para produção de fotocatalisadores heterogêneos ativados por UV-vis é a combinação de materiais, na forma de heteroestruturas, pois pode-se criar um material com regiões ricas em elétrons e outras ricas em buracos. As regiões possuindo alta densidade eletrônica são responsáveis pelas reações de redução, que podem intermediar, por exemplo, a produção de hidrogênio gasoso e outras espécies oxidantes. Já os buracos possuem potencial de oxidação bastante elevado, suficientemente positivo para induzir a produção de radicais $\mathrm{HO}$ a partir de moléculas de água adsorvidas na superfície do semicondutor, as quais induzem a oxidação de um número expressivo de moléculas orgânicas e espécies ionizadas, devido à sua baixa seletividade $[3,4]$. A combinação ou junção de dois ou mais materiais também pode favorecer a transferência interfacial de elétrons excitados para níveis da banda de condução mais próximos (de menor energia), prorrogando sua permanência na banda de condução e diminuindo assim a recombinação com o buraco formado. Desta forma, tanto o processo redutivo quanto o oxidativo são favorecidos.

Compostos bifásicos contendo óxidos dos tipos perovskita e espinélio têm sido estudados devido à coexistência de suas propriedades em um mesmo material heteroestruturado. O principal método de preparação tem sido o de mistura mecânica ou física, no qual os constituintes na forma de pó obtidos separadamente são misturados para produzir um pó aparentemente homogêneo e puro. Ou, mistura de um pó de óxidos do tipo perovskita na solução precursora dos óxidos de espinélio, ou vice-versa. O recente desafio é a preparação simultânea das duas fases numa mesma solução, com controle de formação do compósito bifásico numa única etapa de síntese, denominada in situ, o que até o momento nenhum trabalho na literatura teve esse foco de utilizar a síntese in situ pelo método hidrotérmico para preparar heteroestruturas bifásicas de titanato-ferritas. A produção de um material heteroestruturado que combine a melhoria da propriedade fotocatalítica com a propriedade magnética torna-se atrativo com relação à aplicação, devido a favorecer a posterior separação do catalisador do meio. Entre os novos tipos de materiais heteroestruturados com esse tipo de aplicação e que apresentam boa atividade fotocatalítica têm sido pesquisados $\mathrm{TiO}_{2} / \mathrm{Al}_{2} \mathrm{O}_{3} / \mathrm{NiFe}_{2} \mathrm{O}_{4}$ [5], $\mathrm{TiO}_{2} / \mathrm{NiFe}_{2} \mathrm{O}_{4}[6], \mathrm{Fe}_{3} \mathrm{O}_{4} / \mathrm{NiFe}_{2} \mathrm{O}_{4} / \mathrm{Ni}$ [7], $\mathrm{TiO}_{2}-\mathrm{Al}_{2} \mathrm{O}_{3}-$ $\mathrm{ZnFe}_{2} \mathrm{O}_{4}[8], \mathrm{CoFe}_{2} \mathrm{O}_{4} / \mathrm{CdS}$ [9], $\mathrm{CoFe}_{2} \mathrm{O}_{4}-\mathrm{ZnO}$ [10], $\mathrm{CoFe}_{2} \mathrm{O}_{4}-\mathrm{Fe}_{3} \mathrm{O}_{4}$ [11], $\mathrm{CoFe}_{2} \mathrm{O}_{4}$-grafeno [12], $\mathrm{TiO}_{2} / \mathrm{CoFe}_{2} \mathrm{O}_{4}$ [13] e $\mathrm{CoFe}_{2} \mathrm{O}_{4}-\mathrm{Ag}_{3} \mathrm{PO}_{4}$ [14]. Além desses materiais, o titanato de estrôncio, um semicondutor do tipo n, é citado na literatura como um bom fotocatalisador em substituição ao $\mathrm{TiO}_{2}$ devido à sua alta estabilidade química e fotoquímica, com energia de band gap próxima a $3,2 \mathrm{eV}$ que favorece seu uso como fotocatalisador [15]. O uso combinado do titanato de estrôncio com ferritas na forma de heteroestruturas para a degradação de corantes não é conhecido e assim há também a possibilidade de combinar a propriedade fotocatalisadora com a propriedade magnética das ferritas. Devido à junção desses materiais, pode-se evitar a recombinação do par elétron/buraco levando a fotocatalisadores mais eficientes. Além das propriedades citadas, a produção deste tipo de heteroestruturas, que tenham em sua composição íons de ferro, possibilita também seu uso em processos catalíticos denominados de Fenton, ou no caso de catalisadores fotoativos em processos denominados de foto-Fenton. Desta forma, este trabalho teve como objetivo a síntese in situ de compostos nanométricos heteroestruturados à base de titanato de estrôncio-ferritas utilizando o método hidrotérmico e o estudo das propriedades fotocatalisadoras na degradação do corante Rodamina-B utilizando o processo foto-Fenton.

\section{MATERIAIS E MÉTODOS}

Os pós foram sintetizados in situ utilizando o método hidrotermal utilizando um reator hidrotérmico $(\mathrm{RH})$ com aquecimento em placa convencional, o que possibilitou uma cristalização lenta e homogênea. As quantidades dos reagentes utilizados foram calculadas estequiometricamente para concentrações de 0,005 mols da fase obtida em $120 \mathrm{~mL}$ de solução, sendo a proporção 50/50 para as duas fases. Todos os reagentes utilizados foram de grau analítico. O precursor de titânio foi preparado a partir de uma rota de hidrólise utilizando o RH. Uma suspensão com butóxido de titânio [Ti $\left.(\mathrm{OBu})_{4}\right], 50 \mathrm{~mL}$ de álcool isopropílico, ácido acético glacial (1:1 Ti) e hidróxido de amônio (4:1 Ti), foi levada ao reator a uma temperatura interna de $100{ }^{\circ} \mathrm{C}$, controlada por um termopar, durante $60 \mathrm{~min}$. O precipitado formado foi lavado com água em uma centrífuga, até um $\mathrm{pH}$ de 7 . Em seguida, foram adicionados os nitratos de sais metálicos, sob agitação. Após $15 \mathrm{~min}$, o pH foi reajustado para 13 pela adição de $\mathrm{NaOH} 10 \mathrm{M}$. A solução foi novamente levada ao $\mathrm{RH}$, na placa de aquecimento a $140{ }^{\circ} \mathrm{C}$, com tratamentos térmicos de até $12 \mathrm{~h}$ gerando uma pressão de $3 \mathrm{kgf} / \mathrm{cm}^{3}$. Após o processo, a solução foi coletada à temperatura ambiente $\mathrm{e}$ levada à centrífuga para lavagem e redução do $\mathrm{pH}$ até 7 . Finalmente o precipitado foi levado a uma estufa a $80^{\circ} \mathrm{C} / 12$ $\mathrm{h}$ para secagem, antes de sua caracterização.

Os pós foram caracterizados por difração de raios $\mathrm{X}$ (DRX, Siemens, 5100) com radiação $\mathrm{K} \alpha$ do $\mathrm{Cu}(\lambda=1,5418 \AA)$, operando com tensão de $40 \mathrm{kV}$ e corrente de $40 \mathrm{mAevelocidade}$ do goniômetro de aproximadamente $2 \% \mathrm{~min}$, variando $2 \theta$ de $20^{\circ}$ a $80^{\circ}$. Os difratogramas obtidos foram indexados através das fichas cristalográficas JCPDS-ICDD (Joint Committee on Powder Diffraction Standards - International Center for Diffraction Data). Os espectros de infravermelho das amostras foram obtidos em um espectrômetro Bomem, com resolução de $4 \mathrm{~cm}^{-1}$ na região entre 4000 e $400 \mathrm{~cm}^{-1}$. Para a determinação da área superficial específica, foi utilizado um medidor Gemini-2370, Micromerictis, por meio do método de adsorção de nitrogênio/hélio desenvolvido por Brunauer, 
Emmett e Teller (BET). O tamanho dos pós foi analisado em um microscópio eletrônico de transmissão, Philips CM120, operando com uma aceleração de $120 \mathrm{kV}$. As energias de band gap dos fotocatalisadores foram obtidas por medidas de espectroscopia de reflectância difusa num espectrômetro UV-visível (Shimadzu, UV-2401PC/2501PC) com acessório para reflectância difusa. A atividade fotocatalítica dos compostos foi avaliada através da reação de degradação de Rodamina B, numa solução com concentração de $10^{-5} \mathrm{M}$ de corante, sob agitação magnética e irradiação de luz visível. Os testes fotocatalíticos foram realizados na presença de $\mathrm{H}_{2} \mathrm{O}_{2}$, por $4 \mathrm{~h}$, à temperatura ambiente, na presença de $1 \mathrm{~g} / \mathrm{L}$ de catalisador e uma lâmpada de Xe $200 \mathrm{~W}$ com irradiância de $900 \mathrm{~W} / \mathrm{cm}^{2}$ na região de máxima intensidade $(\sim 350 \mathrm{~nm})$. Alíquotas de $4 \mathrm{~mL}$ da solução foram retiradas a cada 10 min inicialmente e depois a cada hora e levada para análise em um espectrômetro UV-visível. A degradação do corante foi monitorada pela diminuição da banda de absorção do corante em $544 \mathrm{~nm}$.

\section{RESULTADOS E DISCUSSÃO}

As estruturas cristalinas dos compostos heteroestruturados de $\mathrm{SrTiO}_{3}-\mathrm{CoFe}_{2} \mathrm{O}_{4}$ e $\mathrm{SrTiO}_{3}-\mathrm{NiFe}_{2} \mathrm{O}_{4}$ foram confirmadas por difração de raios X (Fig. 1). Os padrões de DRX dos pós sintetizados revelaram que os compostos apresentaram as fases desejadas, obtidas pelo método hidrotérmico in situ. A completa formação das fases aconteceu em função do tempo de síntese, onde foi possível observar a formação de picos bem definidos nas amostras sintetizadas em $12 \mathrm{~h}$. $\mathrm{O}$ tempo de $3 \mathrm{~h}$ não foi suficiente para a total formação das fases de interesse e desta forma a síntese destes compostos em sistema hidrotérmico mostrou-se essencial para a formação das heteroestruturas. Cada pico foi indexado de acordo com fichas padrões, localizadas em bases de dados específicas para DRX em materiais inorgânicos. Os padrões apresentaram picos referentes à fase cúbica do $\mathrm{SrTiO}_{3}-\mathrm{ICSD}^{\circ}$ 86-177, com grupo espacial $P m-3 m$, e picos referentes a fase espinélio cúbico (grupo espacial $\mathrm{Fd} 3 \mathrm{~m}$ ) das ferritas $\mathrm{CoFe}_{2} \mathrm{O}_{4}$ - ICSD no 22-1080, e $\mathrm{NiFe}_{2} \mathrm{O}_{4}$ - ICSD no 89-4927.

Os resultados de espectroscopia na região do infravermelho estão apresentados na Fig. 2. As amostras apresentaram uma banda larga em $3400 \mathrm{~cm}^{-1}$ relativa à presença de grupos hidroxila [16]. Bandas nesta localização caracterizam sítios ativos na superfície do material, com os quais as moléculas adsorvidas fisicamente são unidas por ligações de hidrogênio fracas. Essa banda tem sido observada em outros pós nanocristalinos de titanatos de estrôncio (ST) $[17,18]$. As vibrações na região entre $2000-800 \mathrm{~cm}^{-1}$ estão relacionadas a grupos orgânicos presentes no material ou mesmo na superfície deste. Bandas por volta de $2300 \mathrm{~cm}^{-1}$ são atribuídas à deformação das moléculas de $\mathrm{CO}_{2}$ adsorvidas [19]. As bandas em $1500 \mathrm{~cm}^{-1}$ (mais intensa) e 1067 e $900 \mathrm{~cm}^{-1}$ (menos intensas) são características de grupos carbonatos [20, 21]. Pelos resultados de DRX não foi verificada a presença da fase $\mathrm{SrCO}_{3}$, mas a sua presença pode estar relacionada à presença de grupos hidroxilas na superfície que podem atuar
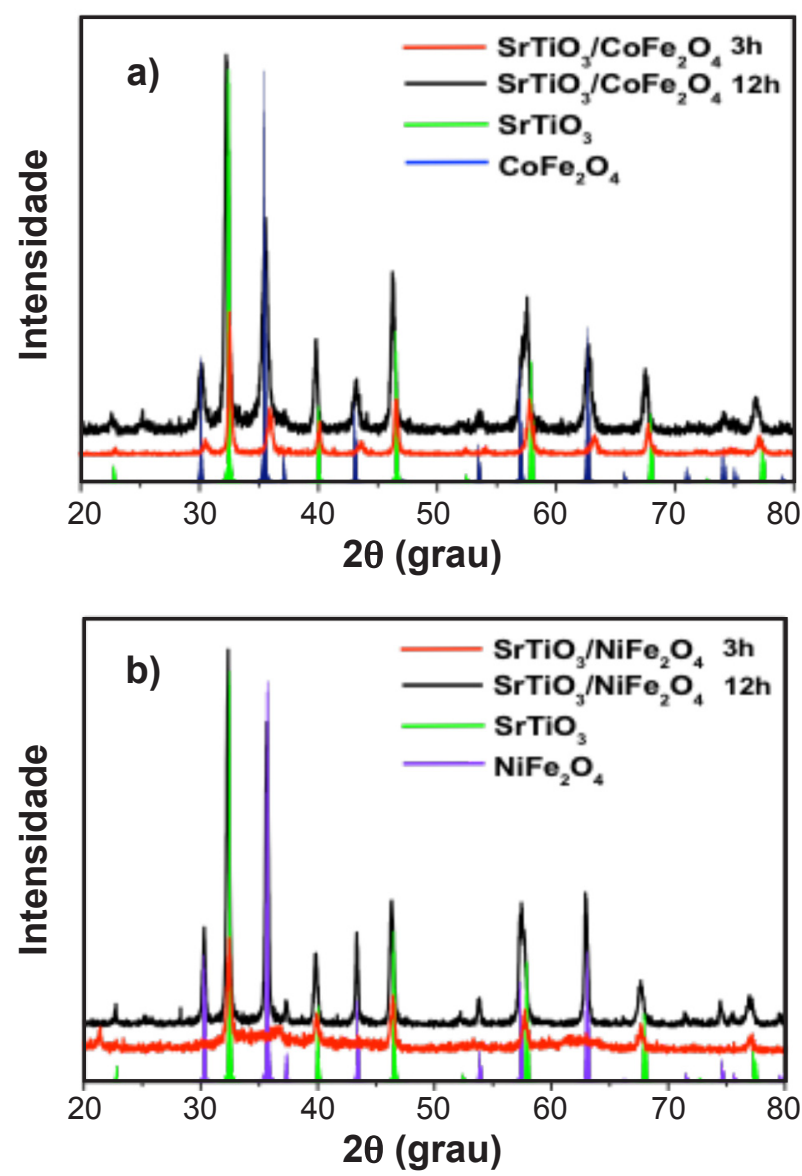

Figura 1: Difratogramas de raios $\mathrm{X}$ das amostras $\mathrm{SrTiO}_{3}-\mathrm{CoFe}_{2} \mathrm{O}_{4}$ (a) e $\mathrm{SrTiO}_{3}-\mathrm{NiFe}_{2} \mathrm{O}_{4}$ (b) sintetizadas in situ pelo método hidrotermal em diferentes tempos de síntese (3 e 12 h).

[Figure 1: X-ray diffraction patterns of samples $\mathrm{SrTiO}_{3}$-CoFe ${ }_{2} \mathrm{O}_{4}$ (a) and $\mathrm{SrTiO}_{3}-\mathrm{NiFe}_{2} \mathrm{O}_{4}$ (b) obtained by in situ hydrothermal synthesis for different lengths of time ( 3 and $12 \mathrm{~h}$ ).]

como centros absorvedores para moléculas de $\mathrm{O}_{2}, \mathrm{CO}_{2}$ e $\mathrm{CO}$ [22]. Por outro lado, vibrações abaixo de $900 \mathrm{~cm}^{-1}$ referemse à interação de íons metálicos com o oxigênio, comuns em compostos inorgânicos. As duas ferritas apresentaram bandas intensas no infravermelho na faixa de $600-550 \mathrm{~cm}^{-1}$, que indicam a vibração dos átomos em sítios tetraédricos, o que pode ser associado à ligação $\mathrm{Fe}-\mathrm{O}$ [23]. Abaixo de $500 \mathrm{~cm}^{-1}$ foi possível observar apenas o início da banda referente à vibração dos átomos em sítios octaédricos, correspondentes às ligações Ni-O e Co-O [24, 25]. No espectro das heteroestruturas e no $\mathrm{SrTiO}_{3}$ puro, uma banda larga entre 800 e 500 $\mathrm{cm}^{-1}$ corresponde a ligações dos metais Sr-O e Ti-O [17].

A partir dos espectros de absorção na região do UV-vis (Fig. 3), foi possível obter o valor do band gap dos materiais e prever a presença de níveis intermediários nesse intervalo de energia, os quais são fundamentais para o favorecimento na resposta fotocatalítica do material quando irradiado com energia correspondente à da luz visível. Como esperado, o $\mathrm{SrTiO}_{3}$ cristalino teve um gap em torno de $3,2 \mathrm{eV}$, calculado a partir do método de Tauc, através do espectro de reflectância difusa [26, 27], e mostrado na Tabela I. O band gap do ST foi indireto e a banda de valência completa 


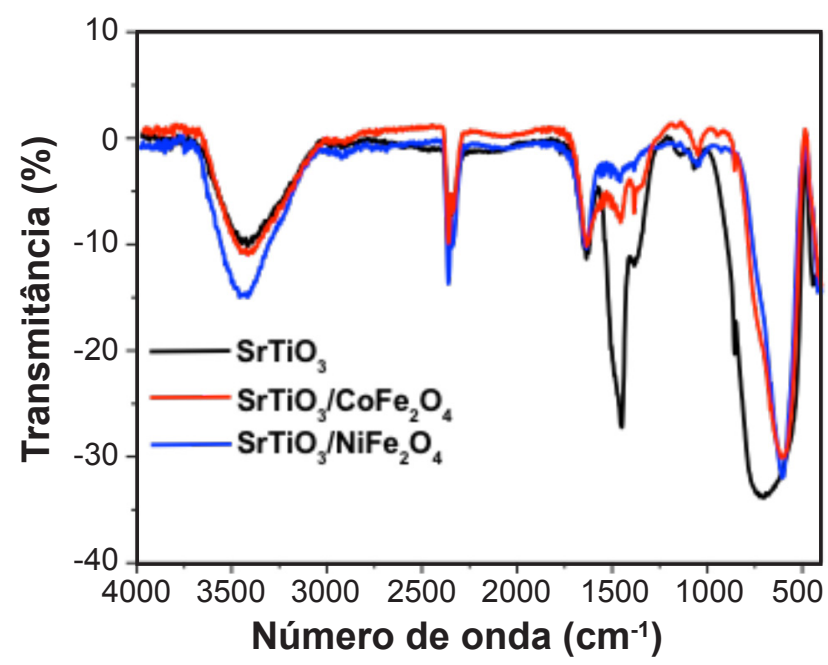

Figura 2: Espectros de absorção na região do infravermelho das amostras $\mathrm{SrTiO}_{3}, \mathrm{SrTiO}_{3} / \mathrm{CoFe}_{2} \mathrm{O}_{4}$ e $\mathrm{SrTiO}_{3} / \mathrm{NiFe}_{2} \mathrm{O}_{4}$.

[Figure 2: Infrared absorption spectra of samples $\mathrm{SrTiO}_{3}, \mathrm{SrTiO}_{3}$ / $\mathrm{CoFe}_{2} \mathrm{O}_{4}$ and $\left.\mathrm{SrTiO}_{3} / \mathrm{NiFe}_{2} \mathrm{O}_{4} \cdot\right]$

derivou dos orbitais $2 p$ do oxigênio e a banda de condução vazia dos orbitais $3 \mathrm{~d}$ do titânio; logo, a borda de absorção observada para este material deveu-se a uma transição de transferência de carga $\mathrm{O}^{2-} \rightarrow \mathrm{Ti}^{4+}$ e o gap foi atribuído à separação energética entre os referidos níveis. Devido à sua banda larga, assim como no óxido de titânio, o ST mostrou uma alta reflectância na região do visível e revelou uma forte absorção apenas em uma pequena faixa espectral da região visível. Por outro lado, com a formação das heteroestruturas de $\mathrm{SrTiO}_{3}-\mathrm{CoFe}_{2} \mathrm{O}_{4}$ e $\mathrm{SrTiO}_{3}-\mathrm{NiFe}_{2} \mathrm{O}_{4}$, a absorção desses compostos notavelmente foi deslocada para região visível, com elevada absorção até $\sim 600 \mathrm{~nm}$. As ferritas de cobalto e as ferritas de níquel são bem estudadas e apresentam de acordo com a literatura um band gap próximo de 1,2 e 1,6 $\mathrm{eV}$, respectivamente $[28,29]$. Assim é possível notar na Fig. 3 que com a formação dessas heteroestruturas os valores de

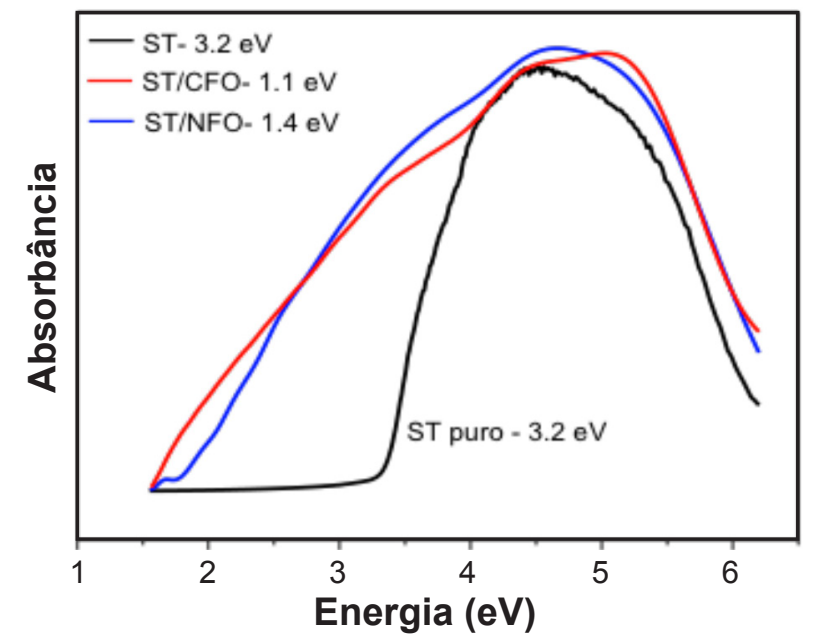

Figura 3: Espectros de absorção na região do UV-visível das amostras $\mathrm{SrTiO}_{3}, \mathrm{SrTiO}_{3}-\mathrm{CoFe}_{2} \mathrm{O}_{4}$ e $\mathrm{SrTiO}_{3}-\mathrm{NiFe}_{2} \mathrm{O}_{4}$.

[Figure 3: UV-vis absorption spectra of samples $\mathrm{SrTiO}_{3}, \mathrm{SrTiO}_{3}$ $\mathrm{CoFe}_{2} \mathrm{O}_{4}$ and $\mathrm{SrTiO}_{3}-\mathrm{NiFe}_{2} \mathrm{O}_{4}$.]
Tabela I - Valores de band gap das amostras sintetizadas in situ pelo método hidrotermal.

[Table I - Band gap values of the samples subjected to in situ hydrothermal synthesis.]

\begin{tabular}{cc}
\hline Composição & Band gap (eV) \\
\hline $\mathrm{SrTiO}_{3}$ & 3,2 \\
$\mathrm{SrTiO}_{3} / \mathrm{NiFe}_{2} \mathrm{O}_{4}$ & 1,4 \\
$\mathrm{SrTiO}_{3} / \mathrm{CoFe}_{2} \mathrm{O}_{4}$ & 1,1 \\
\hline
\end{tabular}

band gap foram ligeiramente diminuídos com relação ao ST puro, o que implica na utilização eficaz desses compostos em reações fotocatalíticas irradiadas com luz visível.

As imagens de microscopia eletrônica de transmissão (MET, Fig. 4) apresentaram partículas nanométricas para os dois compostos, onde as partículas na forma de cubos são relativas à morfologia do titanato de estrôncio $\mathrm{SrTiO}_{3}(\mathrm{STO})$ que estão de acordo com a literatura [30], e as partículas do tipo esféricas, relativas às ferritas. As partículas de $\mathrm{SrTiO}_{3}$ apresentaram tamanhos entre $15-60 \mathrm{~nm}$, e a morfologia do tipo cúbica está de acordo com [31]. Na Fig. 4a é mostrada a formação da heteroestrutura $\mathrm{SrTiO}_{3}-\mathrm{NiFe}_{2} \mathrm{O}_{4}$, onde as partículas em forma de pequenas esferas são relativas à ferrita de níquel $\left(\mathrm{NiFe}_{2} \mathrm{O}_{4}\right)$ com tamanhos de $\sim 20-40 \mathrm{~nm}$ e ao lado é possível observar a composição através da microanálise por EDX. Já as amostras de $\mathrm{SrTiO}_{3}-\mathrm{CoFe}_{2} \mathrm{O}_{4}$, Fig. 4b, apresentaram a formação de aglomerados de partículas nanométricas de $\mathrm{CoFe}_{2} \mathrm{O}_{4}$ na ordem de $\sim 4 \mathrm{~nm}$ ao redor das partículas de titanato de estrôncio. Desta forma, foi possível verificar que o titanato de estrôncio inibiu o crescimento das partículas de ferrita de cobalto, levando a uma textura mais fina e consequentemente a uma maior área de atividade fotocatalítica. Todas as composições foram comprovadas por microanálise. Esses resultados, por sua vez, corroboraram os encontrados nas medidas de área superficial, onde as amostras apresentaram áreas superficiais de $26,2,18,5$ e $40,7 \mathrm{~m}^{2} \cdot \mathrm{g}^{-1}$ para o $\mathrm{SrTiO}_{3}$ puro, $\mathrm{SrTiO}_{3}-$

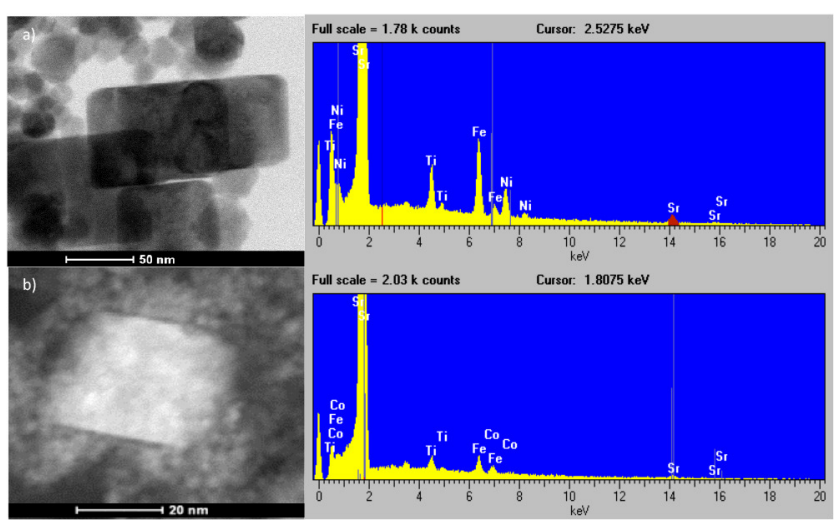

Figura 4: Imagens de MET e espectros de EDX das amostras $\mathrm{SrTiO}_{3}-\mathrm{NiFe}_{2} \mathrm{O}_{4}$ (a) e $\mathrm{SrTiO}_{3}-\mathrm{CoFe}_{2} \mathrm{O}_{4}$ (b) sintetizadas in situ pelo método hidrotermal.

[Figure 4: TEM micrographs and EDX spectra of samples subjected to in situ hydrothermal synthesis: (a) $\mathrm{SrTiO}_{3}-\mathrm{NiFe}_{2} \mathrm{O}_{4}$; and (b) $\mathrm{SrTiO}_{3}-\mathrm{CoFe}_{2} \mathrm{O}_{4}$.] 


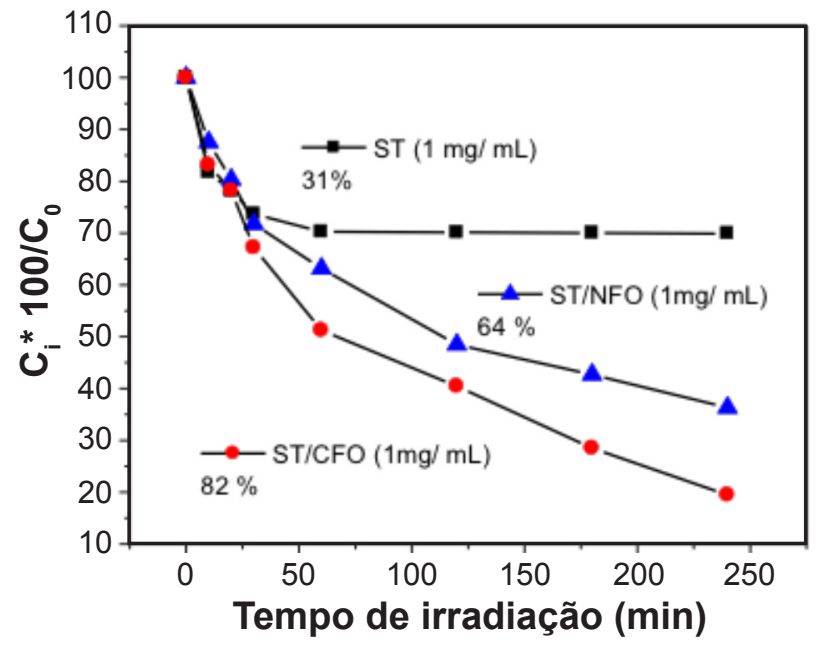

Figura 5: Porcentagem de degradação da $\mathrm{RhB}$ na presença das amostras $\mathrm{SrTiO}_{3}, \mathrm{SrTiO}_{3} / \mathrm{NiFe}_{2} \mathrm{O}_{4}$ e $\mathrm{SrTiO}_{3} / \mathrm{CoFe}_{2} \mathrm{O}_{4}$ em função do tempo de irradiação.

[Figure 5: Percentage of $\mathrm{RhB}$ degradation in the presence of the samples $\mathrm{SrTiO}_{3}, \mathrm{SrTiO}_{3} / \mathrm{NiFe}_{2} \mathrm{O}_{4}$ and $\mathrm{SrTiO}_{3} / \mathrm{CoFe}_{2} \mathrm{O}_{4}$ as a function of irradiation time.]

$\mathrm{NiFe}_{2} \mathrm{O}_{4}$ e $\mathrm{SrTiO}_{3}-\mathrm{CoFe}_{2} \mathrm{O}_{4}$, respectivamente, o que indicou que as partículas do $\mathrm{SrTiO}_{3}-\mathrm{CoFe}_{2} \mathrm{O}_{4}$ foram bem menores em relação aos outros materiais.

As três amostras foram aplicadas na degradação fotocatalítica da Rodamina $\mathrm{B}$ com a adição de $0,5 \mathrm{~mL}$ de $\mathrm{H}_{2} \mathrm{O}_{2}$ e irradiação com luz visível. Os testes catalíticos foram feitos em duplicata. A Fig. 5 apresenta os resultados analisados a partir de espectros de UV-vis, observando-se a diminuição da intensidade do pico referente à concentração do corante Rodamina B em solução, em função do tempo de irradiação. As amostras dos compostos heteroestruturados foram capazes de degradar mais que $50 \%$ do corante, demonstrando assim a importância da estratégia de acoplamento de band gaps, enquanto a amostra pura foi responsável por apenas $31 \%$ de degradação. Após $4 \mathrm{~h}$ de irradiação, a amostra $\mathrm{SrTiO}_{3}-\mathrm{CoFe}_{2} \mathrm{O}_{4}$ apresentou melhores resultados com $82 \%$ de degradação em relação a $64 \%$ do $\mathrm{SrTiO}_{3}-\mathrm{NiFe}_{2} \mathrm{O}_{4}$. O resultado foi satisfatório, visto que o $\mathrm{SrTiO}_{3}-\mathrm{CoFe}_{2} \mathrm{O}_{4}$ apresentou maior área superficial e maior absorção na região do visível, como visto anteriormente, ocasionando um aumento na atividade fotocatalítica.

Os melhores resultados apresentados pela heteroestrutura de $\mathrm{SrTiO}_{3}-\mathrm{CoFe}_{2} \mathrm{O}_{4}$ com relação a $\mathrm{SrTiO}_{3}-\mathrm{NiFe}_{2} \mathrm{O}_{4}$ podem ser atribuídos a uma maior formação de radicais hidroxila. Essa maior formação pode estar atribuída a uma menor recombinação dos pares elétron/buraco e assim as reações de oxidação podem ocorrer entre as lacunas formadas na banda de valência e o peróxido de hidrogênio produzindo mais radicais hidroxila. Já as reações de redução podem ocorrer entre os elétrons da banda de condução e o oxigênio dissolvido na água, produzindo íons radicais superóxidos $\left(\mathrm{O}_{2}^{-}\right)$, os quais podem produzir mais peróxido de hidrogênio, que levam à produção de mais radicais hidroxila, o que está de acordo com [32]. Desta forma, a maior atividade catalítica apresentada pelas heteroestruturas se deve à sinergia devido a um melhor aproveitamento fotônico, visto a diferença de valores de band gaps demonstrados na Tabela I. A recombinação dos elétrons e buracos produz uma diminuição da eficiência quântica [33]. Segundo [34], sabe-se que os semicondutores têm um papel importante em reações de fotocatálise, pois o elétron da banda de condução é aprisionado nessas estruturas na forma de um íon radical superóxido $\left(\mathrm{O}_{2}^{-}\right)$. Assim, a produção de um material com uma heterojunção, como os produzidos neste trabalho, tem a finalidade de diminuir ou impedir a recombinação citada. Desta forma, as heteroestruturas $\left(\mathrm{SrTiO}_{3}-\mathrm{CoFe}_{2} \mathrm{O}_{4}\right.$ e $\mathrm{SrTiO}_{3}-$ $\mathrm{NiFe}_{2} \mathrm{O}_{4}$ ) mostraram-se mais efetivas em relação à atividade fotocatalítica em comparação ao titanato de estrôncio puro $\left(\mathrm{SrTiO}_{3}\right)$.

\section{CONCLUSÕES}

As amostras dos compostos heteroestruturados foram obtidas com sucesso de forma inédita pelo método hidrotérmico in situ, em apenas uma etapa de síntese. A síntese in situ favoreceu a completa formação das fases, com picos de difração de raios $\mathrm{X}$ bem definidos, após 12 $\mathrm{h}$ de tratamento térmico. A estratégia de utilizar ferritas na composição apresentou-se satisfatória, favorecendo ao aumento de absorção de energia no espectro da região do visível, com consequente crescimento de atividade fotocatalítica em relação à fase $\mathrm{SrTiO}_{3}$ pura. $\mathrm{O}$ composto $\mathrm{SrTiO}_{3}-\mathrm{CoFe}_{2} \mathrm{O}_{4}$ apresentou, além de um espectro de absorção na região do UV-visível mais amplo, aglomerados de partículas significativamente menores com elevada área superficial, consequentemente favorecendo a fotodegradação do corante Rodamina B, sob radiação visível.

\section{AGRADECIMENTOS}

Os autores agradecem à Fundação de Amparo à Pesquisa do Estado de São Paulo (FAPESP) pelo apoio financeiro (Processo n. 2008/04025-0), ao Conselho Nacional de Desenvolvimento Científico e Tecnológico (CNPq) pelo apoio financeiro e bolsas concedidas (Processo $\mathrm{n}$. 471441/2013-9) e à Coordenação de Aperfeiçoamento de Pessoal de Nível Superior (CAPES) pela bolsa PNPD (Processo n. 23038.007.008.2011/36) concedida.

\section{REFERÊNCIAS}

[1] C. Camaselle, M. Pazos, M. Sanromán, Chemosphere 60 (2005) 1080.

[2] A.E.H. Machado, L.M.S. Santos, K.A. Borges, P.S. Batista, V.A.B. Paiva, P.S. Muller, D.F. Oliveira, M.D. França, in Solar radiation, InTech, Rijeka (2012) 339.

[3] X. Fan, J. Wan, E.Z. Liu, L. Sun, Y. Hu, H. Li, X. T. Hu, J. Fan, Ceram. Int. 41 (2015) 5107.

[4] A.E.H. Machado, A.O.T. Patrocinio, M.D. França, L.M. Santos, K.A. Borges, L.F. Paula, Mater. Proc. Energy (2013) 867.

[5] M.X. Jiang, C. Han, X.Q. Shen, J. Nanosci. Nanotechnol. 
13 (2013) 4949.

[6] D.D. Tan, D.F. Bi, P.H. Shi, S.H. Xu, Mater. Res. 518 (2012) 775 .

[7] Y. Li, T. Wu, K. Jin, Y. Qian, N. Qian, K. Jiang, W. Wu, G. Tong, Appl. Surf. Sci. 387 (2016) 190.

[8] P.P. Hankare, R.P. Patil, A.V. Jadhav, K.M. Garadkar, R. Sasikala, Appl. Catal. B Environ. 107 (2011) 333.

[9] S. Singh, N. Khare, Mater. Lett. 161 (2015) 64.

[10] A. Franco Jr., H.V.S. Pessoni, F.O. Neto, J. Alloys Compd. 680 (2016) 198.

[11] F. Yi, Ceram. Int. 40 (2014) 7837.

[12] L. Gan, S. Shang, C.W.M. Yuen, S. Jiang, E. Hu, Appl. Surf. Sci. 351 (2015) 140.

[13] P. Sathishkumar, R.V. Mangalaraja, S. Anandan, M. Ashokkumar, Chem. Eng. J. 220 (2013) 302.

[14] L. Gan, L. Xu, K. Qian, Mater. Design 109 (2016) 354. [15] A.N. Pinheiro, E.G.S. Firmiano, A.C. Rabelo, C.J Dalmaschio, E.R. Leite, RSC Adv. 4 (2014) 2029.

[16] S. Adireddy, C.K. Lin, B.B. Cao, W.L. Zhou, G. Caruntu, Chem. Mater. 22 (2010) 19460.

[17] E.R., Leite, C.M.G. Sousa, E. Longo, J.A. Varela, Ceram. Int. 21 (1995) 143.

[18] K. Choi, H. Kil, Y. Lee, D. Lim, S. Cho, B.W. Lee, J. Lumin. 131 (2011) 894.

[19] B. Herreros, T.L. Barr, J. Klinowski, J. Phys. Chem. 98 (1994) 738 .

[20] H.S. Gopalakrishnamurthy, M.S. Rao, T.R.N. Kutty, J. Inorg. Nucl. Chem. 37 (1975) 891.
[21] H. Nishizawa, M. Katsube, J. Solid State Chem. 131 (1997) 43

[22] S.G. Cho, P.F. Johnson, R.A. Condrate, J. Mater. Sci. 25 (1990) 4738.

[23] O.M. Hemeda, J. Magn. Magn. Mater. 28 (2004) 36.

[24] A. Baykal, N. Kasapoğlu, Y. Köseoğlu, S.M. Toprak, H. Bayrakdar, J. Alloys Compd. 464 (2008) 514.

[25] R.D. Waldron, Phys. Rev. 99 (1955) 1727.

[26] M.D. Beals, in High temperature oxide, Vol. 5, Part 2, Ed.: A.M. Alper, Academic Press (1970) 99.

[27] H. Yamamoto, S. Okamoto, H. Kobayashi, J. Lumin. 100 (2002) 325.

[28] B.S. Holinsworth, D. Mazumdar, H. Sims, Q.-C. Sun, M.K. Yurtisigi, S.K. Sarker, A. Gupta, W.H. Butler, J.L. Musfeldt, Appl. Phys. Lett. 103 (2013) 82406.

[29] N.M. Caffrey, D. Fritsch, T. Archer, S. Sanvito, C. Ederer, Phys. Rev. B 87 (2013) 24419.

[30] S. Huang, W.W. Lee, J. Chang, W. Huang, S. Chou, C. Chen, J. Taiwan Inst. Chem. Eng. 45 (2014) 1927.

[31] M. Xu, Y. Lu, Y. Liu, S. Shi, F. Fang, J. Am. Ceram. Soc. 89 (2006) 3631.

[32] R.P.S. Suri, J. Liu, D.W. Hand, J.C. Crittendem, D.L. Perram, M.E. Mullins, Water Environ. Res. 65 (1993) 665.

[33] R.W. Mattheus, Sol. Energy 38 (1987) 405.

[34] K. Okmoto, Y. Yamamoto, M. Tanaka, A. Itaya, Bull. Chem. Soc. Jpn. 58 (1985) 2015.

(Rec. 27/03/2017, Rev. 11/08/2017, 05/10/2017, Ac. $11 / 11 / 2017)$ 